\title{
Diabetic patients' awareness regarding diabetes mellitus and its ocular complications at the National District Hospital in Bloemfontein, Free State, South Africa
}

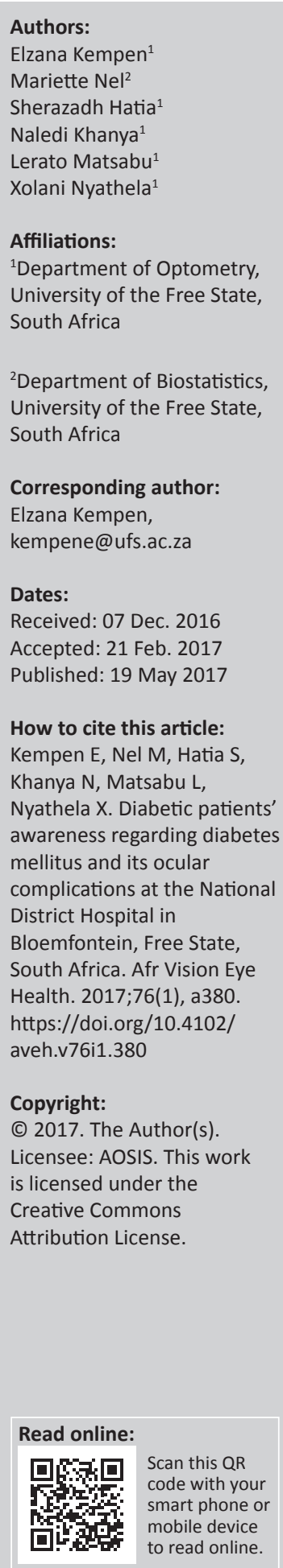

\section{Authors:}

Naledi Khanya ${ }^{1}$

Lerato Matsabu

\section{Affiliations:}

'Department of Optometry, University of the Free State,

${ }^{2}$ Department of Biostatistics, University of the Free State, outh Africa

Corresponding author:

Elzana Kempen,

Dates:

Received: 07 Dec. 2016

Accepted: 21 Feb. 2017

How to cite this article: Kempen E, Nel M, Hatia S, Khanya N, Matsabu L, awareness regarding diabetes mellitus and its ocular complications at the National District Hospital in Health. 2017;76(1), a380. https://doi.org/10.4102/

Copyright:

(c) 2017. The Author(s). Licensee: AOSIS. This work is licensed under the Creative Commons bution License.

\begin{abstract}
Introduction: Globally, the prevalence of diabetes mellitus (DM) and obesity is rapidly increasing. DM and, consequently, diabetic retinopathy (DR) are serious issues that all countries around the world are facing. This study originated from a need to assess whether diabetic patients at the Outpatient Department at the National District Hospital in Bloemfontein have knowledge about their disease and are aware of the possible ocular complications.

Methods: A descriptive study on a single group of participants was conducted at the Outpatient Department at National District Hospital in Bloemfontein. Non-random judgemental sampling was used to select participants who were diagnosed with diabetes. All participants were above the age of 18 and gave signed consent after reading through the information document provided.

Results: The participants $(n=70)$ were mainly women $(72 \%)$ and elderly ( $>40$ years). Age groups represented were 40 years $(7.1 \%), 40-49$ years $(12.9 \%), 50-59$ years $(32.9 \%), 60-69$ years $(28.6 \%)$ and $\geq 70$ years $(18.56 \%)$. Almost $82 \%$ of the participants were aware that DM can cause blindness and $85.7 \%$ were aware of cataracts as a possible complication of DM. Almost half $(47.1 \%)$ were not aware that DM can cause DR and more than half (52.9\%) were unaware that DM can cause increased intraocular pressure (IOP).

Conclusion: These results highlight the need for further education of diabetic patients regarding the relationship between DM and the ocular complications.
\end{abstract}

\section{Introduction}

Diabetes mellitus (DM) can be defined as a group of metabolic diseases, which are characterised by hyperglycaemia resulting from a defect in the secretion of insulin, the function of insulin or both. Chronic hyperglycaemia is associated with long-term damage to various organs, including the eyes and kidneys. ${ }^{1}$ There are two main classifications for DM, namely type I and type II. In type I DM, which is also known as juvenile-onset DM, there is an abnormality with insulin secretion, resulting in insulin deficiency. This may be caused by the destruction of $\beta$-cells of the pancreas or may be idiopathic. Type II DM is seen as the most common form of DM and accounts for more than $90 \%$ of diabetic patients. ${ }^{2}$ In this type of DM, which is also known as adult-onset $\mathrm{DM}$, there is a combination of insulin deficiency due to secretion abnormalities as well as resistance to insulin. Wu et al. ${ }^{2}$ state that there is also a possible genetic correlation in the cause of type II DM. Other influencing factors include a sedentary lifestyle, obesity, unhealthy dietary habits, smoking and alcohol consumption, gut metagenome correlation and a deficiency in vitamin D and K. Systemic symptoms of DM include polydipsia, polyphagia, polyuria and unintentional weight loss, whereas ocular-related symptoms of DM include blurred and fluctuating vision, night blindness as well as flashes and floaters. ${ }^{3}$

Not only is DM a concern in developed countries, but it poses a serious challenge to developing countries such as South Africa. DM is becoming more common as a result of the contributing factors mentioned earlier. ${ }^{3}$ In 2013, Khandekar and Al-Lawati ${ }^{4}$ claimed that 382 million individuals worldwide suffered from DM, and this number is expected to increase to 592 million by the year 2035.

According to the International Diabetes Federation (IDF) Diabetes Atlas ${ }^{5}$, the prevalence of DM in South Africa in 2014 was approximately 8.4\% (2.7 million), with another 1.2 million 
undiagnosed individuals. It is estimated that the South African diabetic population will increase to approximately 3.8 million with the prevalence of DM increasing to approximately $9.8 \%$ by 2035.5

This information is important to optometrists as DM is the third leading cause of blindness in South Africa. ${ }^{5}$ It is, therefore, essential for a patient with DM to have regular eye examinations as DM can cause several complications in the eye such as diabetic retinopathy (DR), including maculopathy, cataracts and glaucoma. Pelino and Pizzimenti ${ }^{3}$ stated that individuals with DM are $40 \%$ more likely to develop glaucoma and $60 \%$ more likely to develop cataracts than individuals without this disease. DR is a condition that affects the eyes of an individual with DM, regardless of whether the DM is type I or type II. It occurs as a result of damage to the blood vessels that nourish the retina, and the longer an individual has DM, the more likely they are to develop some degree of DR. ${ }^{6}$

Approximately $40 \%-45 \%$ of Americans diagnosed with DM have DR. ${ }^{7}$ If untreated, DR may lead to irreversible vision loss and even blindness. ${ }^{4}$ In developed countries, according to Bhavsar, ${ }^{8} \mathrm{DR}$ is responsible for blindness in approximately 8000 eyes yearly, which means that this disease is responsible for $12 \%$ of blindness. Hofman et al. ${ }^{9}$ report that in South Africa, 55\% of individuals diagnosed with DM are likely to have DR. Sukha and Rubin ${ }^{10}$ found in a diabetic clinic in Johannesburg that one in five patients with DM present with DR, whereas Carmichael et al. ${ }^{11}$ found a prevalence of $30 \%$ in their diabetic population. A survey conducted in Cape Town in 2010 found that DR is responsible for $8 \%$ of blindness and $11 \%$ of visual impairment in low- and middle-income communities in South Africa. ${ }^{12}$ In the rural areas of South Africa, the occurrence of DR in DM patients is higher. Rotchford and Rotchford ${ }^{13}$ found an incidence of $40.3 \%$ in their diabetic population in rural KwaZulu-Natal.

$\mathrm{DM}$ and DR are, thus, serious health problems that all countries face, but $\mathrm{Wu}$ et $\mathrm{al} .{ }^{14}$ reported that with proper treatment of DR, more than $90 \%$ of cases of vision loss can be prevented. Therefore, patients with DM must be properly educated on the pathogenesis of the disease and the ocular complications thereof.

Previous studies in South Africa have investigated the knowledge of DM and its ocular implications amongst diabetic patients ${ }^{12,15,16,17,18}$, and Clarke-Farr et al. ${ }^{12}$ found that diabetic patients in the Western Cape had a basic level of understanding of their disease and the possible ocular complications, but noticed that the daily management of the condition was not optimally followed. They further found that only $30 \%$ of these individuals had yearly eye examinations. This agrees with Thomas et al. ${ }^{15}$ who reported that approximately only $11 \%$ of the diabetic population in South Africa undergo routine ocular examinations for DR.

Mashige et al. ${ }^{16}$ conducted a similar study in Durban, South Africa, and stated that the participants took interest in their condition as they were aware of the importance of adjusting their lifestyle and having regular medical evaluations. The participants from the study of Mashige et al. ${ }^{16}$ were mainly from a community Clinic at the University of KwaZulu-Natal's Eye Clinic. Another study, by Van Staden et al., ${ }^{17}$ done in 2015 within KwaZulu-Natal, included participants that attended private and public hospitals. Their results indicated that DM patients from the private sector had generally more knowledge about the disease compared with those from the public sector. Mabaso and Oduntan ${ }^{18}$ conducted a similar study in Limpopo Province, and the results were in agreement with other studies in South Africa, that is, participants had some awareness of $\mathrm{DM}$, but the awareness of its ocular complications was limited.

The current study originated from the need to assess whether diabetic patients in Bloemfontein had knowledge about DM and whether they were aware of its ocular complications. No previous reports could be found on diabetic patients' awareness regarding DM and its ocular complications at the National District Hospital in Bloemfontein, Free State. The study and its results may encourage health care practitioners to educate diabetic patients about the ocular complications that may occur in DM patients and so increase preventative measures to reduce serious visual impairment.

\section{Methodology}

Participants were required to give informed consent before they were included in this study. Consent forms and information documents were available in English, Afrikaans and Sesotho. Participation in this study was voluntary, and participants were informed that they may withdraw from the study at any time. No participants were put at risk for partaking in this study, and the data collected for this study remained confidential.

Aquestionnaire, toassess the participants' general understanding of DM, the complications of the disease and its management, was adapted from a similar study by Clark-Farr et al., ${ }^{12}$ to fit in the current demographics. This questionnaire is available from the authors on request. Structured individual interviews with the participants were conducted in a private room. Nonrandom judgemental sampling was used to select participants diagnosed with diabetes. All participants were above the age of 18 and gave signed consent after reading through the information document provided.

\section{Ethical considerations}

The study was approved by the Health Science Research Ethics Committee of the University of the Free State (STUD NR 68/2015). Permission to collect data from the patients at the Outpatient Department of the National District Hospital was obtained from the Free State Department of Health as well as the CEO of the Outpatient Department. 


\section{Results \\ Demographic profiles}

The participants $(n=70)$ included $71.4 \%$ women and $28.6 \%$ men, with most of the participants speaking Sesotho (52.9\%). The youngest participant was 35 years old, and the oldest was 78 years old. Age groups (years) represented were $<40$ years $(7.1 \% ; n=5), 40-49$ years $(12.9 \% ; n=9), 50-59$ years $(32.9 \% ; n=23), 60-69$ years $(28.6 \% ; n=20)$ and $\geq 70$ years $(18.6 \% ; n=13)$. More than half of the participants $(71.4 \%)$ were diagnosed with DM less than 15 years ago, with the remaining participants (28.6\%) diagnosed 15 years or more ago. Only $5.7 \%(n=4)$ knew that they were diagnosed with type $1 \mathrm{DM}$, whereas $20 \%(n=14)$ were diagnosed with type 2 DM. The rest of the participants $(74.3 \% ; n=52)$ did not know which type of DM they presented with.

\section{General knowledge of diabetes}

Most of the participants (62.9\%) were diagnosed with diabetes by a general practitioner (GP), with a community clinic being the second most common source of diagnosis (28.6\%). Most of the participants $(60 \%)$ reported that they go for regular check-

TABLE 1: Participant responses regarding the importance of different factors in controlling diabetes at the Outpatient Department at the National District Hospital in Bloemfontein, South Africa.

\begin{tabular}{lccc}
\hline $\begin{array}{l}\text { Are the following factors } \\
\text { important in controlling your } \\
\text { diabetes? }\end{array}$ & Yes (\%) & No (\%) & Do not know (\%) \\
\hline Diet & 95.7 & 2.9 & 1.4 \\
Exercise & 100.0 & - & - \\
Blood glucose level & 95.7 & 4.3 & - \\
Body weight & 95.7 & 2.9 & 1.4 \\
Taking medication regularly & 98.6 & - & 1.4 \\
Routine medical check-ups & 94.3 & 4.3 & 1.4 \\
\hline
\end{tabular}

$N=70$. ups regarding their health; however, many (51.4\%) were not aware that two types of DM exist, and the majority (74.3\%) did not know which type they had. A large percentage of the participants (61.4\%) thought that DM could be inherited, and $12.9 \%$ did not know whether it is a genetic condition or not. Most of the participants (64.3\%) knew that having DM for a longer time increases your chances of developing complications as a result thereof. Table 1 indicates the responses of importance regarding different factors in controlling diabetes. With regard to the importance of lifestyle changes, participants were most aware of the importance of exercising (100\%) and controlling of blood glucose levels (95.7\%). Overall, more than half (57.1\%) of the participants believed that they had sufficient knowledge regarding the management of DM.

\section{Ocular knowledge of diabetes}

Less than half (41.4\%) of the participants were advised to have regular eye examinations when they were initially diagnosed. Most of the participants (64.3\%) reported that they did not go for regular eye examinations. More than half $(n=47)$ of the participants reported that they had either never had an eye test or had last had an eye examination $>2$ years ago. Despite the finding that few participants went for regular eye examinations, a large portion (87.2\%) considered regular eye examinations important. The majority of the participants were aware that DM can cause blindness ( $81.4 \%$ ) and cataracts $(85.7 \%)$, whereas almost half (47.1\%) were unaware that DM can cause DR and more than half $(52.7 \%)$ were unaware that DM can cause increased intraocular pressure (IOP) (see Figure 1).

Most of the participants (70\%) reported that they were satisfied with the amount of information they had received from their health care worker regarding the complications of

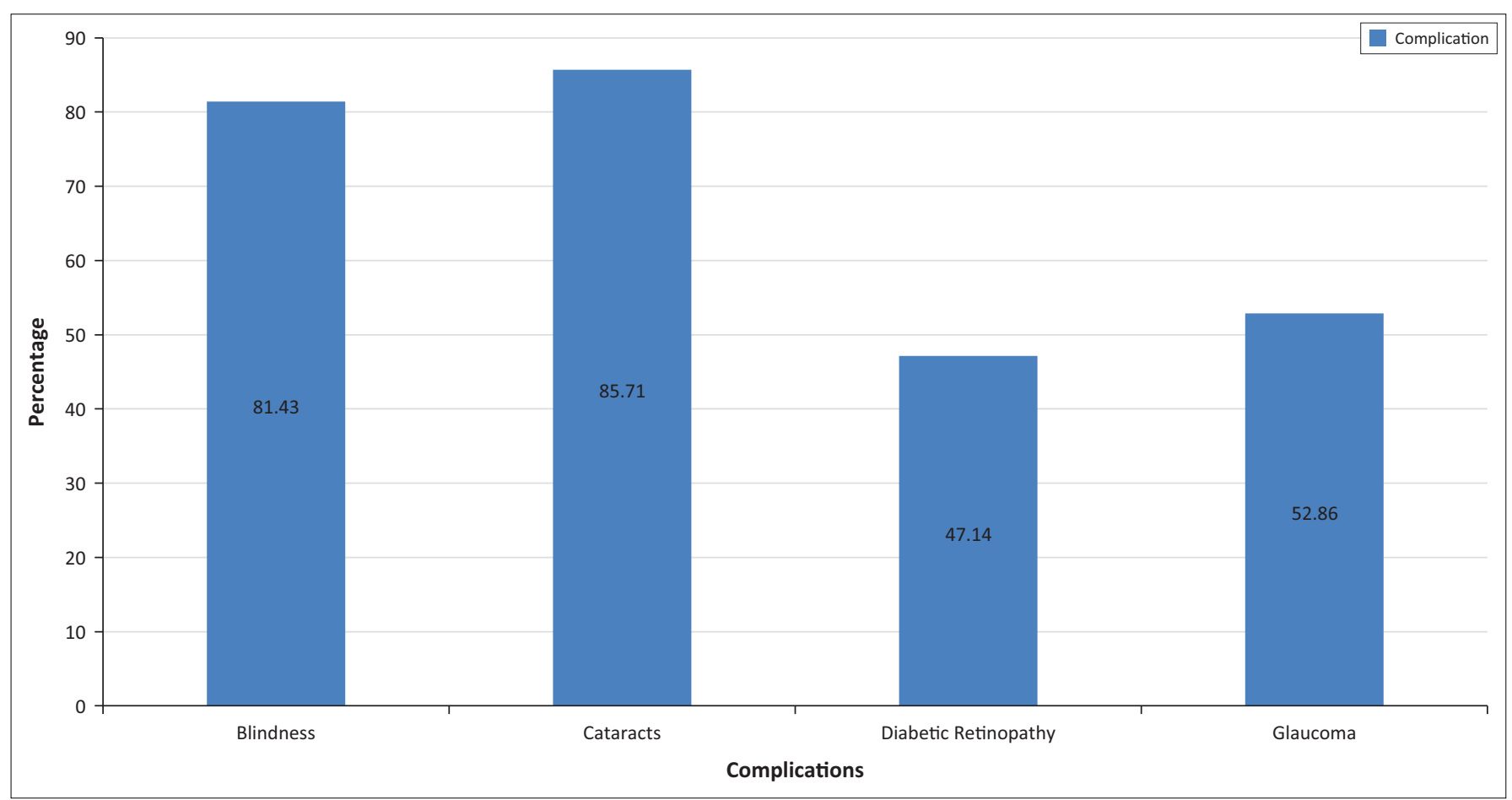

FIGURE 1: Awareness of ocular complications of diabetes mellitus amongst 70 diabetic patients at the National District Hospital in Bloemfontein, South Africa. 
DM on the eye, but most of the participants (71.4\%) chose to receive additional information regarding DM in the form of pamphlets.

\section{Discussion}

Of the 70 participants, more than half were women which may indicate that either most men with this condition are undiagnosed or perhaps go elsewhere for medical treatment, or that women in Bloemfontein are at two times greater risk of developing this condition as compared with men. This finding is consistent with other studies done in South Africa. $12,15,16,17,18$ In the current study, the most represented age group of the participants were $50-59$ years (32.8\%) with only $7.1 \%$ of the participants younger than 40 years of age. Mabaso and Oduntan ${ }^{18}$ explained that the increased age of the participants might be due to older people being more likely to utilise government health services than the younger age groups.

GPs continue to play a decisive and vitally important role as primary care givers in Bloemfontein as about $63 \%$ of the participants were diagnosed with DM by such medical professionals compared to nurses, ophthalmologists or optometrists. More than half of the participants interviewed were unaware of the two types of DM (type I and type II) with almost $75 \%$ unaware of which type they had. The limited knowledge on the type of DM that a participant may have is of great concern as this information is necessary to better their understanding and knowledge of the condition and consequently the management thereof. A contributing factor may include that GPs do not inform their newly or diagnosed patients upon consultations but instead assume that this piece of information is actually common knowledge or that GPs do not consider it as important information for newly diagnosed diabetics or that patients themselves are not doing enough to acquire information about DM.

All (100\%) participants were aware of the importance of exercise, whereas approximately $96 \%$ of them understood the importance of monitoring their glucose levels. It must be noted that even though all participants were aware of the importance of exercise, this does not necessarily indicate that all were physically active individuals.

The results revealed that upon diagnosis, almost half (42\%) of diagnosed diabetics were not informed of the need for routine ocular examinations. This is a possible explanation as to why optometrists note the presence of DM-related ocular complications often in its latter stages, once vision starts to deteriorate or when the individual becomes visually impaired. The results showed that about $65 \%$ of participants did not have regular eye examinations and a quarter never had the opportunity to go for an eye examination. The question here is, do patients diagnosed with diabetes really understand the severity of their condition and the possible impact thereof on their eyes? Or is it merely that the population in Bloemfontein is not sufficiently educated regarding DM?
Furthermore, more than half of the participants were unaware that DM can cause increased IOP but over $80 \%$ claimed that they were aware of complications such as blindness and cataracts. This supports the statement made earlier that optometrists may be faced with consulting such patients only when their condition has severely progressed.

In contrast to the above-mentioned figures and statements, $70 \%$ of the participants claimed to be satisfied with the level of information provided to them by their health care worker, which gives rise to questions such as whether we really are providing our patients with relevant information in a language they understand and whether patients take the information provided to them seriously or do patients simply not understand what is being explained to them regarding $\mathrm{DM}$, the disease itself as well as the possible complications?

\section{Conclusion}

In conclusion, we recommend that future studies be done on this same topic, however, with a greater sample size over a wider geographical region. Furthermore, in future studies of people diagnosed with diabetes, the focus should be aimed at GPs, optometrists and ophthalmologists, with the objective of evaluating the information that health practitioners provide to their patients. Further studies may include the language in which patients are communicated with and how health professionals overcome communication barriers to convey relevant information to patients. More insight should be gained as to why individuals with DM are not going for regular eye examinations, despite reporting that they consider them important. Some of the issues here may relate to financial constraints, difficulty accessing suitable sites or individuals with the necessary skills in some regions; or patient fears or ideas concerning possible complications of their condition. This suggested research may aid in providing further insight into issues regarding possible deficiencies in patient education and knowledge of DM and its complications and management.

\section{Acknowledgements Competing interests}

The authors declare that they have no financial or personal relationships that may have inappropriately influenced them in writing this article.

\section{Authors' contributions}

E.K. provided leadership on the student project, with coresearchers being S.H., N.K., L.M. and X.N. who together conducted the fieldwork and compiled the initial draft of this article. M.N. guided the analysis of the data. E.K. guided the write up of the data as well as compiled the final article.

\section{References}

1. American Diabetes Association (ADA). Diagnosis and classification of diabetes mellitus. Diabetes Care. 2010;33(1): S62-S69. 
2. Wu Y, Ding Y, Tanaka Y, Zhang W. Risk factors contributing to Type 2 diabetes and recent advances in the treatment and prevention. Int J Med Sci. 2014:11(11):11851200. https://doi.org/10.7150/ijms.10001

3. Pelino CJ, Pizzimenti JJ. Contemporary care protocols for DR and DME [homepage on the Internet]. 2012 [cited 2015 Jun 06]. Available from: http://www. reviewofoptometry.com/continuing_education/tabviewtest/lessoned/108494

4. Khandekar R, Al-Lawati J. Epidemiology of diabetic retinopathy in Oman: Two decades of research. Oman J Ophthalmol. 2015;8(1):1-2. https://doi. org/10.4103/0974-620X.149853

5. International Diabetes Federation (IDF). Diabetes Atlas, sixth edition [homepage on the Internet]. 2014 [cited 2015 Jun 10]. Available from: http://www. diabetesatlas.org/

6. American Optometric Association (AOA). Diabetic retinopathy [homepage on the Internet]. 2014 [cited 2015 May 30]. Available from: http://www.aoa.org/ patients-and-public/eye-and-vision-problems/glossary-of-eye-and-visionconditions/diabetic-retinopathy?sso=y

7. National Eye Institute. Facts about diabetic eye disease [homepage on the Internet]. 2015 [cited 2015 Jun 06]. Available from: https://nei.nih.gov/health/ diabetic/retinopathy

8. Bhavsar AR. Diabetic retinopathy [homepage on the Internet]. 2015 [cited 2015 Jun 06]. Available from: http://emedicine.medscape.com/article/1225122overview

9. Hofman KJ, Cook C, Levitt N. Preventing diabetic blindness: A priority for South Africa. S Afr Med J. 2014;104(10):661-662. https://doi.org/10.7196/SAMJ.8580

10. Sukha AY, Rubin A. Demographic, medical and visual aspects of Diabetic Retinopathy (DR) and Diabetic Macular Edema (DME) in South African diabetic patients. S Afr Optom. 2009;68(2):70-81. https://doi.org/10.4102/aveh.v68i2.157
11. Carmichael TR, Carp GI, Welsh ND, Kalk WJ. Effective and accurate screening for diabetic retinopathy using $60^{\circ}$ mydriatic fundus camera. S Afr Med J. 2005:95:57-61. https://doi.org/10.1080/22201009.2005.10872109

12. Clarke-Farr $P$, Nel $M$, Wilkinson A. An investigation into diabetic patients' knowledge of diabetes mellitus and its ocular complications in the Western Cape. S Afr Optom. 2006;65(4):134-143.

13. Rotchford AP, Rotchford KM. Diabetes in rural South Africa - An assessment of care and complications. S Afr Med J. 2002;92(7):536-541.

14. Wu L, Fernandez-Loaiza P, Sauma J, Hernandez-Bogantes E, Masis M. Classification of diabetic retinopathy and diabetic macular edema. World J Diabetes 2013;4(6):290-294. https://doi.org/10.4239/wjd.v4.i6.290

15. Thomas RL, Distiller L, Luzlo SD, Chowdhury SR, Melville VJ, Kramer B. Ethnic differences in the prevalence of diabetic retinopathy in persons with diabetes when first presenting at a diabetes clinic in South Africa. Diabetes Care. 2013;36(2):336-341. https://doi.org/10.2337/dc12-0683

16. Mashige KP, Notshweleka A, Moodley S, et al. An assessment of the level of diabetic patients' knowledge of diabetes mellitus, its complications and management in Durban, South Africa. S Afr Optom. 2008;67(3):95-105. https:// doi.org/10.4102/aveh.v67i3.190

17. Van Staden D, Deutshamann LJ, Ganas S, et al. Knowledge of diabetes mellitus and its ocular complications amongst diabetic patients attending private and public its ocular complications amongst diabetic patients attending private and public Vision Eye Health. 2015;74(1). https://doi.org/10.4102/aveh.v74i1.36

18. Mabaso RG, Oduntan OA. Knowledge and practices related to diabetes mellitus among adults with diabetes in the Mopani district, Limpopo Province, South Africa. African Vision Eye Health [serial online]. 2016 [cited 2016 July 16];75(1). Available from: http://avehjournal.org/index.php/aveh/article/ view/324 